\title{
- ๑ SILAE: Nuevas perspectivas en investigación
}




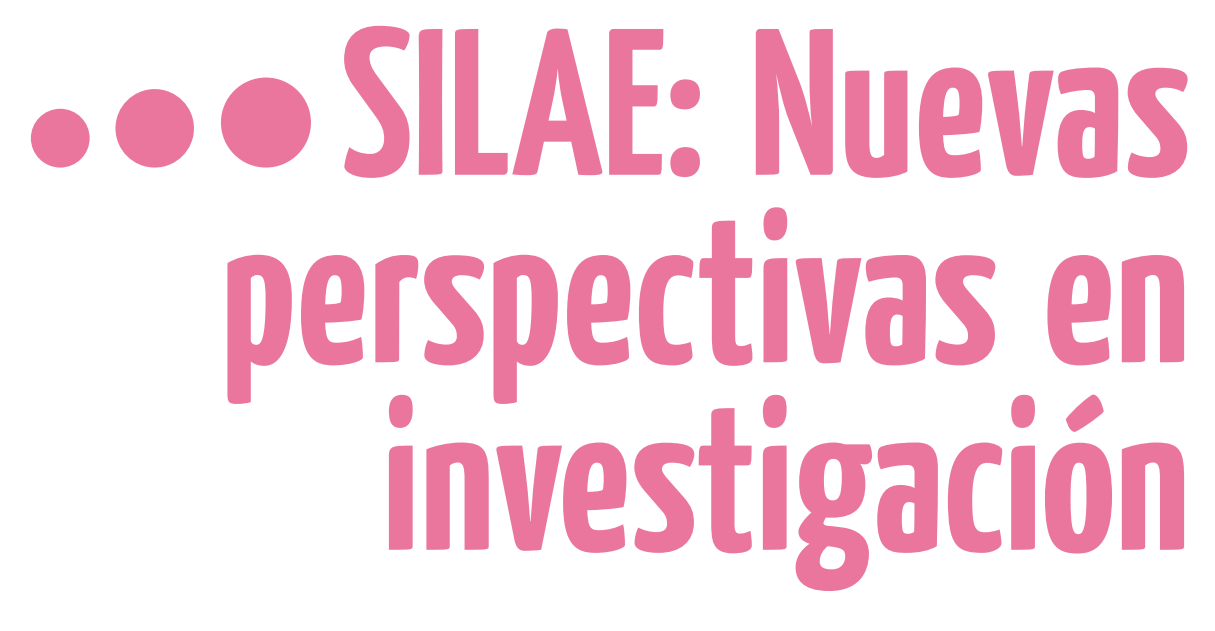

Presentaciones Orales 


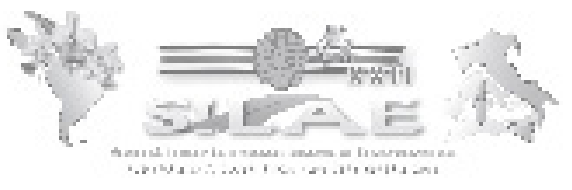

\title{
APPLICATION OF PHASE TRAFFICKING METHODS TO NATURAL PRODUCTS RESEARCH: A NOVEL TOOL FOR BIOPROSPECTING
}

\author{
Juan J. Araya, Barbara Timmermann, Lester Mitscher \\ Department of Medicinal Chemistry, University of Kansas, Lawrence, KS, 66045, USA.
}

Solid supported reagents (SSR) are widely employed in synthetic chemistry, particularly in combinatorial organic synthesis, allowing for quick and easy purification of desired compounds without requiring extensive sample handling. Despite the multiple advantages of SSR for the isolation of small organic molecules from mixtures, this method has yet to find application in resolving natural product extracts. We have, therefore, designed and optimized an application that takes advantage of weak ion exchange resins for a simultaneous rapid recovery of basic, neutral and acidic natural components from plant crude organic extracts. Since spatially separated resins do not interfere with each other's functions, we physically confined basic and acidic resins into "tea bags" followed by their immersion in solutions of plant extracts and optimized recovery of acidic, neutral and basic compounds. The separated phenolics/carboxylic acids, neutrals and alkaloid-enriched fractions, were then recovered from the respective resin bags and the neutral components were recovered from the processed solution. This novel approach offers multiple advantages over traditional isolation methods such as liquid/liquid partition protocols, as it is not labor intensive, makes use of only small quantities of "green" solvents, can be easily adapted to field conditions for bioprospecting, and does not require specialized equipment. We envision that this new method could be applied more widely to natural extracts of diverse origin in order to generate better quality samples for initial bioassays by increasing the relative concentration of desired compounds and reducing interference from undesirable components in the mixtures. The utility of the methodology will be illustrated by presentation of results obtained with artificial mixtures and with extracts of Camellia sinensis (L.) Kuntze and Skytanthus acutus Meyen. 


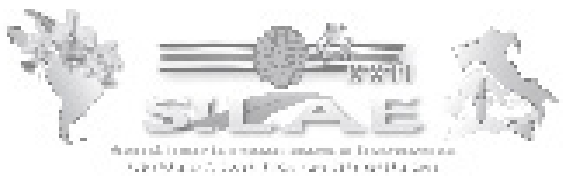

\title{
BIOACTIVES FROM NATURAL SOURCES EXTRACTED USING GREEN PROCESSES
}

\author{
M. Herrero, J.A. Mendiola, M. Castro-Puyana, A. Cifuentes, E. Ibáñez \\ Instituto de Investigación en Ciencias de la Alimentación CIAL (CSIC-UAM). C/Nicolás Cabrera 9, Campus de Cantoblanco, \\ 28049 Madrid, España. elena@ifi.csic.es
}

At present, there is an enormous interest in finding new bioactive compounds able to prevent or improve the health status of the individuals, mainly acting as food supplements, nutraceuticals or functional food ingredients. Undoubtedly, nature can be considered an unlimited source of bioactives and the search of new compounds with improved activities have ran parallel to the search for new natural sources. Different families of compounds have been extracted from plants, although nowadays there is a huge interest in the potential use of marine natural sources to obtain these bioactives, mainly considering their huge diversity, in terms of number of different species that might be potentially used, their sometimes unique chemical structures and their ability to work as natural bioreactors potentiating the synthesis of valuable compounds depending on the cultivation conditions.

Moreover, researchers are facing new challenges in the optimization of new extraction processes to obtain bioactives from natural sources: the development of fast, selective, efficient, sustainable, green (without using toxic organic solvents), with high yields and at lower cost extraction processes is a must. The techniques able to meet these requirements are, among others, those based on the use of compressed fluids such as supercritical fluid extraction (SFE), pressurized liquid extraction (PLE) and subcritical water extraction (SWE), which are among the more promising processes $[1,2]$. In this presentation, different examples will be shown, considering different raw materials such as plants, algae and food by-products and employing these sustainable green technologies. With this approach we will try to demonstrate the possibility of tuning the extraction conditions depending on the target compound(s) and the raw material.

\section{ACKNOWLEDGMENTS}

This work was financed thanks to AGL2011-29857-C03-01 (Ministerio de Economía y Competitividad) and ALIBIRD, S2009/AGR-1469 (Comunidad de Madrid) projects. M.C.P. thanks MICINN for her "Juan de la Cierva" contract. M.H. would like to thank MICINN for a "Ramón y Cajal" research contract.

\section{REFERENCES}

1. King, M.B. and Bott, T.R. Extraction of natural products using near-critical solvents. 1993, Glasgow: Blackie Academic \& Professional.

2. Mendiola, J.A., Herrero, M., Cifuentes, A., Ibáñez, E. Use of compressed fluids for sample preparation: Food applications. Journal of Chromatography A, 2007. 1152(1-2): p. 234-246. 


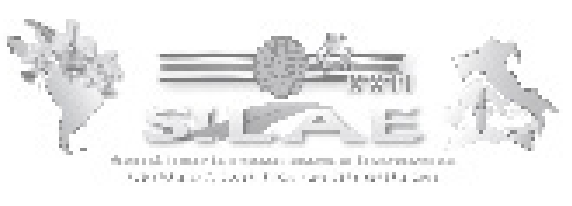

Presentación Oral>SILAE: Nuevas Perspectivas de Investigación

\title{
I NUTRACEUTICI: FARMACI PER PERSONE SANE
}

\author{
Ettore Novellino
}

Department of Medicinal Chemistry, University of Naples "Federico II

Siamo tutti convinti di essere persone sane? Cos'è oggi la salute? Siamo soliti associare il termine farmaco nel caso di patologie e in seguito alla diagnosi, che speriamo venga fatta correttamente, attendiamo l'effetto di queste molecole introdotte nel nostro organismo con la speranza di guarire. Facendo riferimento ad una declaratoria dell'OMS del 1947 concernente il concetto di salute era riportato: "La salute è un completo stato di benessere fisico mentale e sociale e non soltanto l'assenza di malattia". Se riflettiamo possiamo immaginare che nell'immediato periodo postbellico quello non poteva che essere un auspicio a cui si doveva tendere. A partire dagli anni '50 la società è stata oggetto di quattro rivoluzioni repentine e significative. La rivoluzione industriale ha permesso a gran parte delle famiglie di avere un reddito certo a fine mese e quindi di poter programmare la propria vita; la rivoluzione alimentare ha dato possibilità di poter accedere a cibi svariati di prima qualità rispetto a quelli che erano i cibi contingenti e quindi di bassa qualità. La rivoluzione scolastica ha consentito a tutti di poter accedere a quelle che sono le informazioni di ogni genere e con l'ausilio di internet tutto è disponibile nella misura di microsecondi. Nel 1978 con l'istituzione del SSN ci si poneva come obiettivo il mantenimento e il recupero della salute fisica e psichica di tutta la popolazione senza distinzione di condizioni individuali o sociali e secondo modalità che assicurino l'eguaglianza dei cittadini. Tutte queste azioni hanno migliorato notevolmente le condizioni di vita raggiungendo un risultato che nessun attuariale poteva immaginare. Dal 1970 al 2000 abbiamo avuto un incremento di vita di 15 anni; nel 1970 la vita media in Italia che è stato sempre uno dei paesi più longevi era di 65 anni, nel 2000 questa stessa vita media era di 80 anni. Realizzando oggi ciò che l'OMS si poneva di fare non solo siamo riusciti ad avere un completo benessere fisico, mentale e sociale con l'assenza della malattia ma abbiamo aggiunto quello che possiamo definire il concetto di "bell'essere"; d'altronde la società accetta e premia, al primo impatto, un aspetto piacente facendo nascere un grande conflitto tra l'età anagrafica e l'età biologica. leri la società chiedeva di aggiungere anni alla vita oggi chiede di aggiungere vita agli anni. Vorremmo chiedere ai farmaci di farci oltrepassare gli effetti che appartengono alla fisiologia del tempo che passa introducendo la medicina preventiva e quelli che definiamo "Lifestyle drugs" indicati per il trattamento dei fattori di rischio che derivano da stili di vita impropri, o da condizioni, non esclusivamente di tipo patologico, che limitano il benessere di un individuo. Alla medicina di attesa è stata aggiunta la medicina di iniziativa. Introdurre nel nostro organismo un giusto apporto di vitamine, proteine e quant'altro importanti per l'accrescimento e mantenimento di meccanismi fisiologici preesistenti risulta essere sempre più difficile in quanto non è facile seguire una sana e corretta alimentazione. Fondatore e presidente della Foundation for Innovation in Medicine, Stephen De Felice, medico, nel 1989 a Roma, conia per primo il termine nutraceutical da "nutrition" e "pharmaceutical". Secondo la definizione originale, il nutraceutico può essere ritenuto, "un alimento (o parte dell'alimento) che dà effetti benefici alla salute (principio medicamentoso), inclusa la prevenzione e/o il trattamento di una malattia". Quindi è la crasi tra "alimento-farmaco" che associa all'alta digeribilità e all'ipoallergenicità le proprietà curative di principi attivi naturali di comprovata e riconosciuta efficacia. Vi sono potenziali usi terapeutici per malattie dell'apparato gastrointestinale, malattie dismetaboliche e del sistema cardiovascolare, patologie oculistiche, patologie della prostata, menopausa e artrosi cronica. In quest'area di studio e applicazioni ricadono gli alimenti funzionali e gli integratori alimentari. Secondo I' Art. 2 D.L. n 16921 Maggio 2004 si tratta di alimenti caratterizzati da effetti addizionali dovuti alla presenza di componenti naturalmente presenti o aggiunti che interagiscono più o meno selettivamente con una o più funzioni fisiologiche dell'organismo portando ad effetti positivi sul mantenimento della salute e/o prevenzione delle malattie. Gli yogurt, ad esempio, contengono fitosteroli ad azione ipocolesterolemizzante o peptidi ad attività ipotensiva e gli alimenti a base di proteine di soia, anche questi ipocolesterolemizzanti. Ovviamente l'assunzione di tali sostanze può avere benefici per un range limitato di persone che possono rimandare l'assunzione di farmaci indicati dalla medicina tradizionale in questo caso statine. Studi recenti hanno dimostrato che il cioccolato amaro contiene polifenoli che sarebbero in grado di ridurre la pressione arteriosa e dilatare le arterie coronariche (epicatechine). Per integratori alimentari si intendono prodotti destinati ad integrare la comune dieta e che costituiscono una fonte concentrata di sostanze nutritive, quali vitamine e minerali, o di altre sostanze aventi un effetto nutritivo o fisiologico, in particolare ma non in via esclusiva aminoacidi, acidi grassi essenziali, fibre ed estratti di origine vegetale, sia monocomposti che pluricomposti, in 
forme predosate. Di notevole importanza tra gli integratori alimentari risultano essere i probiotici: supplementi alimentari microbici vivi in grado di migliorare la funzione intestinale. Tuttavia l'attore principale del mercato è proprio il consumatore che deve effettuare una scelta consapevole. A sua tutela interviene la legislazione europea avvalendosi di un ente specifico in materia, la European Food Safety Authority (EFSA).

Il crescente interesse per la scienza e del consumatore per gli integratori alimentari e gli alimenti funzionali nasce dalla crescente consapevolezza degli effetti collaterali dei farmaci e delle loro controindicazioni. Con il miglioramento delle condizioni di vita diventano più frequenti alcune patologie come diabete, malattie cardiovascolari e obesità con le annesse complicanze. La nutraceutica sembra avere notevoli possibilità di sviluppo nonostante gli innumerevoli controlli di qualità sulla composizione e sul contenuto dei principi attivi che devono essere effettuati per dimostrare un certo valore salutistico. Scienziati e professionisti della nutrizione stanno facendo grandi sforzi per progettare prodotti sempre migliori e secondo le Autorità di Sanità Pubblica tali prodotti se consumati nell'ambito di una sana e corretta alimentazione rappresentano un utile complemento nutrizionale. Urge un intervento consapevole e maturo di noi farmacisti in questo settore ancora poco analizzato poiché dovremo essere in grado di soddisfare le richieste in tal senso dei consumatori, con la competenza che tradizionalmente accompagna il nostro operato. Con pazienza ed attenzione potremo finalmente riempire di senso la frase di lppocrate: "Lascia che il cibo sia la tua medicina. 